\title{
Moderate Intensity Exercise Eliminates the Inflammation Marker in the Liver of High-Fat-Diet- Induced Obese Mice
}

\author{
Pipit Pitriani* \\ Faculty of Sports and Health Education \\ Universitas Pendidikan Indonesia \\ Bandung, Indonesia \\ *pipitpitriani@upi.edu
}

\author{
Hee-Geun Park, Wang-Lok Lee \\ Department of Sports Science, College of Natural Science \\ Chungnam National University \\ Daejeon, South Korea \\ leewl@cnu.ac.kr
}

\begin{abstract}
The obese metabolic complication has already occurred nowadays. The purpose of the study was to investigate the effect of moderate intensity exercise on inflammation marker in the liver of high fat-diet-induced-obese mice. The 4 weeks male C57BL/6 mice were randomly dispersed to three groups: normal diet control $(\mathrm{NC} ; \mathrm{n}=10)$, high-fat diet control $(\mathrm{HC} ; \mathrm{n}=10)$, a high fat diet with moderate intensity exercise (HME; $n=10)$ groups. The high fat diet was given $60 \%$ calories from fat whereas normal diet was given $18 \%$ calories from fat. The moderate intensity exercise group (HME) was set at $10 \mathrm{~m} / \mathrm{min}$ in the first 2 weeks, $12 \mathrm{~m} / \mathrm{min}$ in $3-5$ weeks and $14 \mathrm{~m} / \mathrm{min}$ in $6-16$ weeks. The inflammation markers were checked by quantitative Real Time PCR. The body and liver weight was significantly increased in the high fat diet group. The moderate intensity exercise significantly reduced the gene expression of TNF $\alpha$, and IL-1 $\beta$ the pro inflammatory cytokine IL-10 was significantly decreased in the high fat diet group but not with moderate intensity exercise. In conclusion, moderate intensity exercise has a positive effect on the liver of high-fat-diet-induced obese by reducing inflammation gene expressions. However, there was no effect of moderate intensity exercise on TLR4 and IL-10.
\end{abstract}

Keywords—exercise; inflammation marker; obesity

\section{INTRODUCTION}

The obese metabolic complication has already occurred nowadays. The obesity-associated state of chronic low-grade systemic inflammation is a proinflammatory condition in which hypertrophied adipocytes and adipose tissue-resident immune cells, both contribute to increased circulating levels of proinflammatory cytokines [1,2]. This proses not only occur in adipose tissue but also in a liver cell that promotes the nonalcoholic fatty liver disease [3].

The fatty liver was formed by accretion of hepatic lipid from enhanced free fatty acids (FFA) influx and de novo lipogenesis [4-7]. The FFA generates oxygen free radicals (OFR) in the mitochondrial chain that causing lipid peroxidation and induces pro-inflammatory cytokine synthesis due to Kupffer cells and hepatocytes, such as tumor necrosis factor-alpha (TNF- $\alpha$ ), transforming growth factor beta-1 (TGF$\beta 1$ ), FAS ligand, and interleukin-8 (IL-8) [7].
Exercise increases energy expenditure and burns off some of the body fat that would otherwise accumulate in individuals who consume more dietary energy than they need, therefore, exercise reduces the risk of developing obesity and excessive adiposity [8]. Current evidence supports that exercise training, such as aerobic and resistance exercise, reduces chronic inflammation, especially in obese individuals with high levels of inflammatory biomarkers undergoing a longer-term intervention $[9,10]$.

Peripheral adipose tissue and hepatic insulin resistance were diminished in weight loss whereas regular exercise changes the development of fatty liver disease $[4,11,12]$. Aerobic-exercise-training ameliorates liver fat accumulation in mice without instigating any changes in adipose tissue mass $[11,13]$. Furthermore, the hepatic TNF- $\alpha$, F $/ 80$, chemokine expression and hepatic fibrosis markers in the liver attenuate by exercise training [13].

Since exercise has a good effect on inflammation in obesity especially adipose tissue, it will be good understanding the effect of exercise in the liver. Therefore, the purpose of this study is to investigate the effects of moderate intensity exercise on inflammation marker in the liver of high fat diet induced obese mice.

\section{MethOD}

This experimental study using four weeks old male C57BL/6 mice, $\mathrm{n}=60$ were bought from the Central Experimental Animal, Korea. After one-week adaptation period, the mice fed by either a high fat diet $(60 \%$ of calories from fat, $20 \%$ from carbohydrate, $20 \%$ from protein, Orient Bio Inc., \#D12492) or a normal diet (18\% calories from fat, $58 \%$ from carbohydrate, $24 \%$ from protein, Orient Bio Inc., \#2018) ad libitum for 16 weeks. They were caged by 5 -miceper-cage with standard experimental laboratory, at temperature $22 \pm 2^{\circ} \mathrm{C}$, and $60 \pm 5 \%$ humidity. The mice weighed the body weight and food intake per cage (5 mice) every week. The calorie intake is calculated from the total food intake conversion to calories. The mice were randomly assigned to 3 groups: normal diet control (NC; $\mathrm{n}=10)$, high-fat diet control 
$(\mathrm{HC} ; \mathrm{n}=10)$ and e high-fat diet with moderate intensity exercise (HME; $\mathrm{n}=10$ ).

Exercise training was initiated from the first week until the sixteenth week that consists of two type intensity: moderate and high-intensity exercise. For moderate-intensity exercise group (HME) the running speed was set at $10 \mathrm{~m} / \mathrm{min}$ for the first 2 weeks, $12 \mathrm{~m} / \mathrm{min}$ for $3-5$ weeks and $14 \mathrm{~m} / \mathrm{min}$ for $6-16$ weeks. The exercise training mice were exercised for 40-60 $\mathrm{min} /$ day and 4 days/week on a treadmill. During exercise, the control group also exposed the same environmental stresses from treadmill noise and vibration. External stimulation or electrical shock not allowed, in order to minimize stress during exercise.

All the mice were sacrificed after fasted for 12 hours under anesthesia using a mixture of ketamine $(80 \mathrm{mg} / \mathrm{kg})$ and xylazine $(10 \mathrm{mg} / \mathrm{kg})$. Before analysis, the tissues of liver were removed, weighed, frozen in liquid nitrogen, and stored at $-70^{\circ} \mathrm{C}$.

Total RNA was extracted from $50 \mathrm{gr}$ liver tissue homogenate using $1 \mathrm{ml}$ Trizol reagent (Ambion, Carlsbad, CA, USA). The total RNA concentration was calculated by measuring the absorbance at $260 \mathrm{~nm}$ and $280 \mathrm{~nm}$ using an ultraviolet spectrophotometer. For cDNA synthesis, Maxi RT PreMix kit (iNtRON, Korea) was used according to the manufacturer's instructions. Polymerase Chain Reaction (PCR) was performed with the CFX 96 touch real-time PCR detection system using the Smobio ExcelTaq 2X Q-PCR Master Mixed. Glyceraldehyde-3-phosphate dehydrogenase (GAPDH) mRNA was used as the housekeeping gene, and all data represented relative to its expression.

Statistical analysis was performed by SPSS V22.0 using One-way ANOVA with LSD posthoc tests. Statistical significance was defined as $\alpha=0.05$.

\section{RESULTS AND DISCUSSION}

The study result of calorie intake was shown in Figure 1. The calorie intake was significantly increased in high-fat diet control group with respect to normal diet control group. However, the high-fat diet with moderate intensity exercise group was not significantly different with respect to the highfat diet control group.

High-fat diets used to induce obese contain $32 \%$ to $60 \%$ of calories from fat. Other study reported that diets with $60 \mathrm{kcal} \%$ fat are frequently used to induce obesity in rodents [14,15]. There are some mice carry a genetic trait that predisposes them to store fat when dietary fat content is high such as the C57BL/6 mice [16].

In a recent study, we used a normal diet with $18 \%$ of calories from fat and high-fat diet with $60 \%$ of calories from fat for 15 weeks. A recent study found there was not significantly different in the high fat diet with moderate intensity group. This founding is contrary with the previous study that mentioned increasing exercise intensity leads to an increase in calorie intake during the meal that follows the exercise session [17]. The differences in methods among these studies, particularly exercise intensity, nutritional status, gender, macronutrient composition of test foods and time intervals between exercise and eating affect these inconsistencies $[18,19]$.

The study result in body weight was shown in Figure 2. We found that the body weight of C57BL/6 mice was significantly increased in the high-fat diet control group. Exercise is able to change energy balance in short to medium period however in the absence of energy restriction, long-term exercise affects the only a small amount of weight loss [20-25]. Moderateintensity exercise without weight loss or calorie restriction is associated with substantial reductions in total and visceral fat, and skeletal muscle lipid with a significant improvement in fitness levels in obese individuals [26]. Some physiological and metabolic process may explain the lack of exercise effectiveness as a weight-loss strategy, first, exercise affects changes in body composition by reducing fat mass but maintaining fat-free mass, second, exercise is related with a decrease in energy expended in basal metabolic rate as a result of weight loss [24].

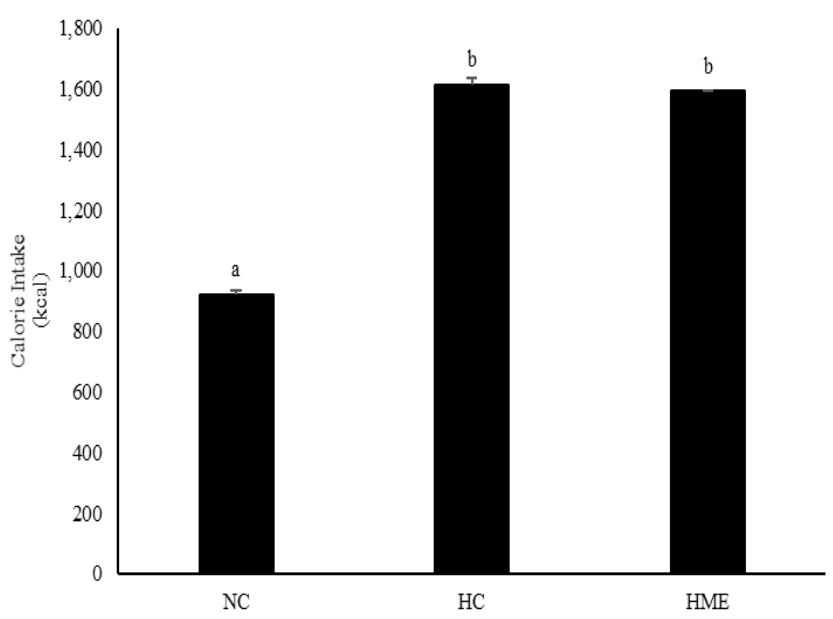

Fig. 1. Effect of moderate intensity exercise on the calorie intake of high fat diet induced obese mice. Data represent means \pm SE. Different alphabet means significant difference.

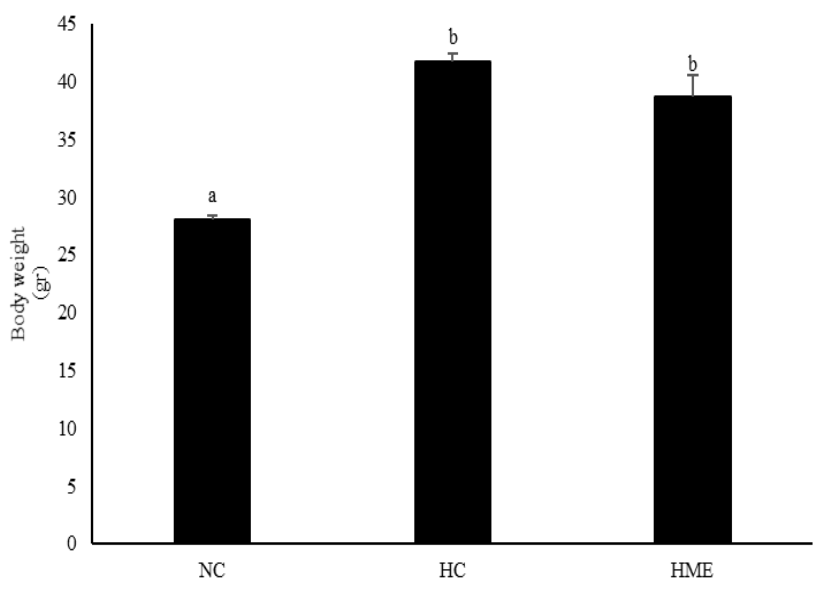

Fig. 2. Effect of moderate intensity exercise on body weight of high fat diet induced obese mice. Data represent means \pm SE. Different alphabet means significant difference. 
The study result in liver weight was shown in Figure 3. The liver weight of the high-fat diet control group was significantly increased with respect to the normal diet control group $(\mathrm{p}=0.00)$. However, the liver weight of a high-fat diet with moderate-intensity exercise groups was not significantly different with respect to the high-fat diet control group.

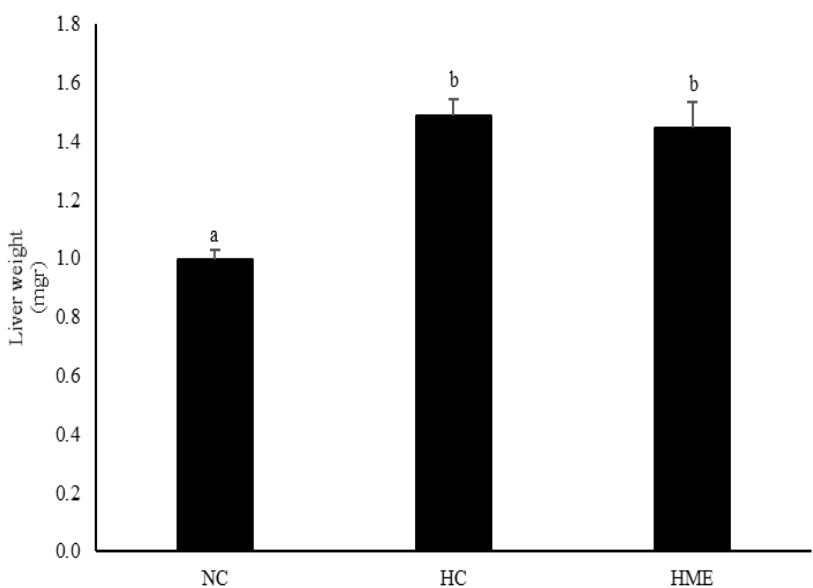

Fig. 3. Effect of moderate intensity exercise on the liver weight of high fat diet induced obese mice. Data represent means \pm SE. Different alphabet means significant difference.

Application of lifestyle interventions, such as caloric restriction and exercise, is highly recommended for the treatment of obesity and associated metabolic dysfunction [9]. The anti-inflammatory effects of exercise have been established [8,10,27]. High fat diet induced obesity metabolic inflammation in the adipose tissue leads to inflammation in the liver [28]. Tumor necrosis factor- $\alpha(\mathrm{TNF} \alpha)$ is an inflammatory cytokine involved in liver inflammation and sustained liver inflammation leads to liver fibrosis. TNF $\alpha$ exerts inflammation, proliferation, and apoptosis [29].

The study result of TNF $\alpha$ gene expression was shown in Figure 4. The TNF $\alpha$ gene expression of high-fat diet control was significantly increased with respect to the normal diet control group $(\mathrm{p}<0.01)$. The gene expression of $\mathrm{TNF} \alpha$ was significantly decreased in the high-fat diet with moderate intensity exercise $(\mathrm{p}<0.05)$ with respect to the high-fat diet control group. This result was similar to our previous study that mentioned moderate intensity exercise ameliorate the TNF- $\alpha$ [30-32]. Moderate intensity exercise decreases TNF- $\alpha$ mRNA manifestation in adipose tissue and liver obese mice $[33,34]$. TNF- $\alpha$ have a special character in the growth of NASH by alluring hepatic fibrosis and injury [35]. Therefore, moderate exercise training may overturn TNF- $\alpha$, which is a specific marker for NASH due to high fat diet-induced obesity.

The study result of IL-1 $\beta$ gene expression was shown in Figure 5. The IL-1 $\beta$ gene expression of high-fat diet control was significantly increased with respect to the normal diet control group $(\mathrm{p}=0.00)$. The gene expression of $\mathrm{IL}-1 \beta$ was significantly decreased in the high-fat diet with moderate intensity exercise group $(\mathrm{p}=0.00)$ with respect to the high-fat diet control group.

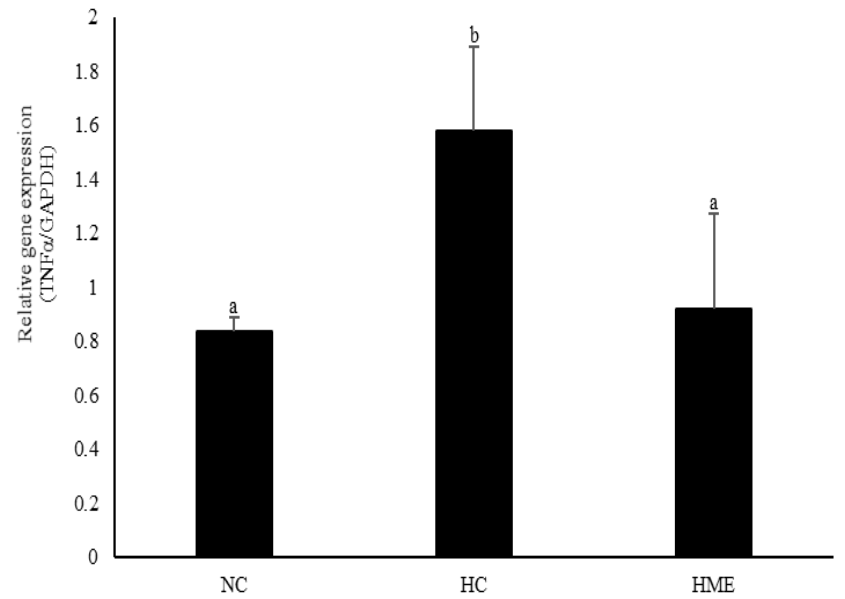

Fig. 4. Effect of moderate intensity exercise on inflammation marker TNFa in high fat diet induced obese mice. Data represent means \pm SE. Different alphabet means significant difference.

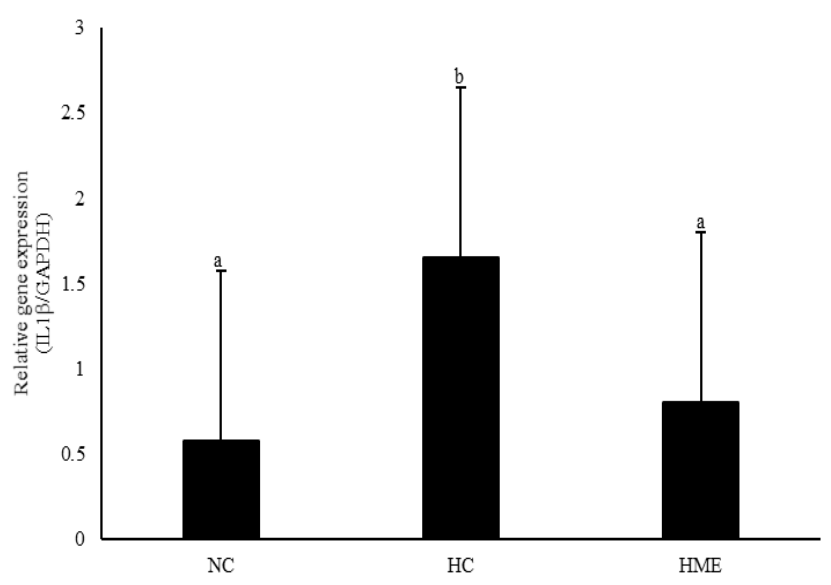

Fig. 5. Effect of moderate intensity exercise on IL1 $\beta$ in high fat diet induced obese mice. Data represent means \pm SE. Different alphabet means significant difference.

The study result of TLR4 gene expression was shown in Figure 6. The TLR4 gene expression was not significantly increased in high-fat diet control group with respect to normal diet control group $(\mathrm{p}=0.794)$. However, the high-fat diet with moderate intensity exercise was significantly reduced the TLR4 gene expression with respect to the high-fat diet control group. Regular exercise acts as anti-inflammatory agent by regulating immune TLR4 cell [36]. 


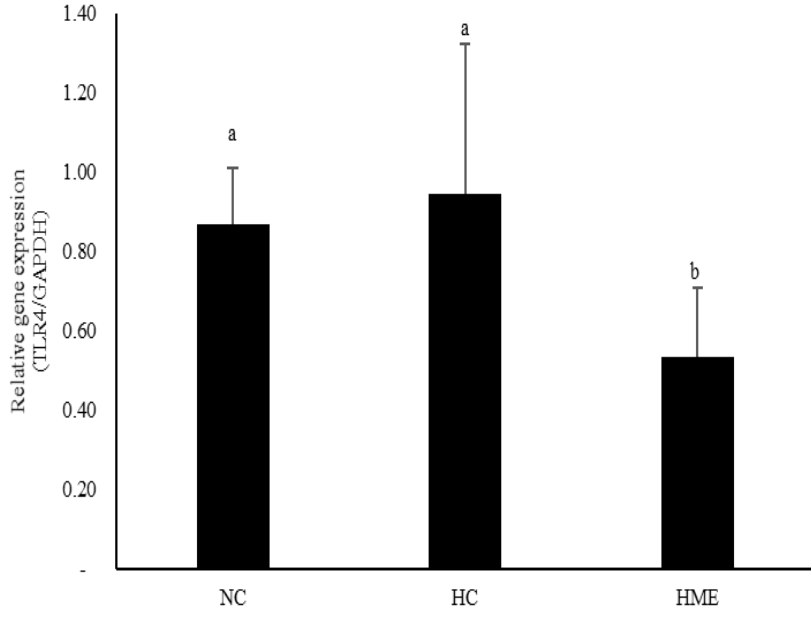

Fig. 6. Effect of moderate intensity exercise on TLR4 in high fat diet induced obese mice. Data represent means \pm SE. Different alphabet means significant difference.

The study result of IL-10 gene expression was shown in Figure 7. The IL-10 gene expression of high-fat diet control was significantly reduced with respect to the normal diet control group $(\mathrm{p}=0.00)$. Gene expression of the antiinflammatory cytokine IL-10 was persistently elevated in HFD mice as well [28]. However, the high-fat diet with moderate intensity exercise was not significantly increased the IL-10 gene expression with respect to the high-fat diet control group.

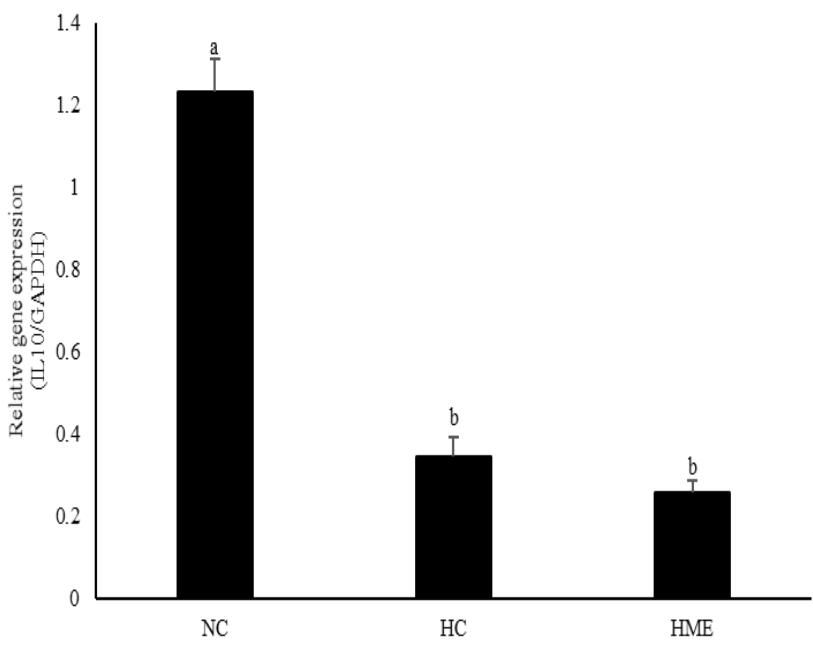

Fig. 7. Effect of moderate intensity exercise on IL-10 in high fat diet induced obese mice. Data represent means \pm SE. Different alphabet means significant difference.

IL-10 is anti-inflammatory cytokine inflammation normalize the physiological or pathological conditions by inhibiting the function of $\mathrm{T}$ cell, monocyte, and macrophagemediated in several organs and tissues [37]. IL-10 has been distinguished in some liver cells, including hepatocytes, stellate cells, and Kupffer cells, but only partial research that has been accomplished to explore the role of endogenous IL-10 in the progress of NAFLD. Other study suggested that endogenous IL-10 was defensive for hepatic steatosis, but not for attendant insulin resistance in IL10 deficient mice [38]. Another study detected that the inhibition of IL-10 increased the expression of IL-1 $\beta$, F4/8, TNF- $\alpha$, IL-6, and reduced insulin signal transduction and steatosis [39]. Our study found that moderate intensity exercise in high-fat-diet-induced obese mice decreased the mRNA expression of IL-10.

\section{CONCLUSION}

Obesity-induced metabolic inflammation in the AT precedes inflammation in the liver, suggesting that the liver does not play a role in the initial development of metabolic inflammation. Modification lifestyle such as increasing physical activity and decreasing fat consumption was already recommended. Furthermore, we show that moderate intensity exercise can eliminate the inflammation marker TNF $\alpha$ and IL$1 \beta$. However, it not enough to decrease the TLR4 and increase the IL-10.

\section{ACKNOWLEDGMENT}

"This work was supported by the National Research Foundation of Korea Grant funded by the Korean Government (NRF-2013S1A5B5A07048323)".

\section{REFERENCES}

[1] K. Makki, P. Froguel, and I. Wolowczuk, "Adipose Tissue in ObesityRelated Inflammation and Insulin Resistance: Cells, Cytokines, and Chemokines," ISRN Inflamm., vol. 2013, pp. 1-12, 2013.

[2] C.N. Lumeng and A.R. Saltiel, "Review series Inflammatory links between obesity and metabolic disease," Life Sci., vol. 121, no. 6, pp. 2111-2117, 2011

[3] Y. Koyama, D.A. Brenner, Y. Koyama, and D.A. Brenner, "Liver inflammation and fibrosis Find the latest version: Liver inflammation and fibrosis," vol. 127, no. 1, pp. 55-64, 2017.

[4] P. Dietrich and C. Hellerbrand, "Non-alcoholic fatty liver disease, obesity and the metabolic syndrome," Best Pract. Res. Clin. Gastroenterol., vol. 28, no. 4, pp. 637-653, 2014.

[5] J.K. Dowman, J.W. Tomlinson, and P.N. Newsome, "Pathogenesis of non-alcoholic fatty liver disease," Qjm, vol. 103, no. 2, pp. 71-83, 2010.

[6] E. Fabbrini, S. Sullivan, and S. Klein, "Obesity and nonalcoholic fatty liver disease: Biochemical, metabolic, and clinical implications," Hepatology, vol. 51, no. 2, pp. 679-689, 2010

[7] V. Martín-Domínguez, R. González-Casas, J. Mendoza-JiménezRidruejo, L. García-Buey, and R. Moreno-Otero, "Pathogenesis, diagnosis and treatment of non-alcoholic fatty liver disease.," Rev. Española Enfermedades Dig., vol. 105, no. 7, pp. 409-20, 2013.

[8] M. Gleeson, N.C. Bishop, D.J. Stensel, M.R. Lindley, S.S. Mastana, and M.A. Nimmo, "The anti-inflammatory effects of exercise : mechanisms and implications for the prevention and treatment of disease," Immunology, vol. 11, pp. 1-10, 2011.

[9] T. You, N.C. Arsenis, B.L. Disanzo, and M.J. LaMonte, "Effects of Exercise Training on Chronic Inflammation in Obesity Current Evidence and Potential Mechanisms," Sport. Med, 2013.

[10] N.P. Walsh, M. Gleeson, R.J. Shephard, M.G. Jeffrey, A. Woods, N.C Bishop, M. Fleshner, C. Green, K. Pedersen, L. Hoffman-goetz, and C.J. Rogers, "Part one: Immune function and exercise," Exerc. Immunol. Rev., vol. 17, pp. 6-63, 2011.

[11] C.M.M. Marques, V.F. Motta, T.S. Torres, and M.B. Aguila, "Beneficia effects of exercise training ( treadmill ) on insulin resistance and nonalcoholic fatty liver disease in high-fat fed C57BL / 6 mice Beneficial effects of exercise training ( treadmill ) on insulin resistance 
and nonalcoholic fatty liver diseas," Brazilian J. Med. Biol. Res., vol. 43, no. 5, pp. 467-475, 2010.

[12] C. Thoma, C.P. Day, and M.I. Trenell, "Lifestyle interventions for the treatment of non-alcoholic fatty liver disease in adults: A systematic review," J. Hepatol., vol. 56, no. 1, pp. 255-266, 2012.

[13] S. Alex, A. Boss, A. Heerschap, and S. Kersten, "Exercise training improves liver steatosis in mice.," Nutr. Metab. (Lond)., vol. 12, no. 29, pp. 1-14, 2015.

[14] L. Ghibaudi, J. Cook, C. Farley, M. van Heek, and J.J. Hwa, "Fat intake affects adiposity, comorbidity factors, and energy metabolism of sprague-dawley rats.," Obes. Res., vol. 10, no. 9, pp. 956-63, 2002.

[15] S.L. Johnston, D.M. Souter, B.J. Tolkamp, IJ. Gordon, A.W. Illius, I. Kyriazakis, and J.R. Speakman, "Intake compensates for resting metabolic rate variation in female C57BL/6J mice fed high-fat diets," Obesity (Silver Spring)., vol. 15, no. 3, pp. 600-606, 2007.

[16] R.S. Surwit, M.N. Feinglos, J. Rodin, A. Sutherland, A.E. Petro, E.C. Opara, C.M. Kuhn, and M. Rebuffe-Scribe, "Differential effects of fat and sucrose on body composition in C57BL/6 and $\mathrm{A} / \mathrm{J}$ mice.," Metabolism., vol. 47, no. 11, pp. 1354-1359, 1998

[17] M. Pomerleau, P. Imbeault, T. Parker, and E. Doucet, "Effects of exercise intensity on food intake and appetite in women," Am. J. Clin. Nutr., vol. 80, no. 5, pp. 1230-1236, 2004

[18] T. Yoshikawa and S. Fujimoto, "Association of exercise with appetite and energy intake through endocrine mechanism," J. Phys. Fit. Sport. Med., vol. 1, no. 2, pp. 211-217, 2012.

[19] J.A. Douglas, J.A. King, D.J. Clayton, A.P. Jackson, J.A. Sargeant, A.E. Thackray, M.J. Davies, and D.J. Stensel, "Acute effects of exercise on appetite, ad libitum energy intake and appetite-regulatory hormones in lean and overweight/obese men and women," Int. J. Obes., vol. 41, no. 12, pp. 1737-1744, 2017.

[20] V.A. Catenacci, "The role of physical activity in producing and maintaining weight loss," Nat Clin Pr. Endocrinol Metab., vol. 3, no. 7, pp. 518-529, 2007.

[21] M.J. Franz, J.J. VanWormer, A.L. Crain, J.L. Boucher, T. Histon, W. Caplan, J.D. Bowman, and N.P. Pronk, " $\{$ A figure is presented $\}$ WeightLoss Outcomes: A Systematic Review and Meta-Analysis of WeightLoss Clinical Trials with a Minimum 1-Year Follow-Up,” J. Am. Diet. Assoc., vol. 107, no. 10, pp. 1755-1767, 2007.

[22] A.S. Greenberg and M.S. Obin, "Obesity and the role of adipose tissue in inflammation and metabolism," Am J Clin Nutr, vol. 83, no. 2, p. 461S-465, 2006.

[23] M. Headland, P.M. Clifton, S. Carter, and J.B. Keogh, "Weight-loss outcomes: A systematic review and meta-analysis of intermittent energy restriction trials lasting a minimum of 6 months," Nutrients, vol. 8, no. 6, 2016.

[24] C. Martins, L. Morgan, and H. Truby, "A review of the effects of exercise on appetite regulation: an obesity perspective," Int. J. Obes., vol. 32, no. 9, pp. 1337-1347, 2008 .

[25] P. Wiklund, "The role of physical activity and exercise in obesity and weight management: Time for critical appraisal," J. Sport Heal. Sci., vol. 5, no. 2, pp. 151-154, 2016.

[26] S. Lee, J.L. Kuk, L.E. Davidson, R. Hudson, K. Kilpatrick, T.E. Graham, R. Ross, J.L. Kuk, L.E. Davidson, K. Kilpatrick, and T.E. Graham, "Role of Exercise in Reducing the Risk of Diabetes and Obesity Exercise without weight loss is an effective strategy for obesity reduction in obese individuals with and without Type 2 diabetes," vol. 6 , pp. 1220-1225, 2005 .

[27] F.M. Steckling, F.D. Lima, J. Boufleur, D. Lopes, D. Santos, and F Alexandre, "Obesity , Inflammation and Aerobic Physical Exercise," Ann Sport. Med Res, vol. 2, no. 2, p. 1017, 2015.

[28] A. Van Der Heijden, F. Sheedfar, M.C. Morrison, P.P.H. Hommelberg, N.J. Kloosterhuis, N. Gruben, S.A. Youssef, A. De Bruin, H. Marten, R. Kleemann, D.P.Y. Koonen, and P. Heeringa, "High-fat diet induced obesity primes inflammation in adipose tissue prior to liver in C57BL/6j mice," Aging (Albany. NY)., vol. 7, no. 4, pp. 256-267, 2015.

[29] Y.M.Y. and E. Seki, "TNF $\alpha$ in liver fibrosis," Curr Pathobiol Rep., vol. 3 , no. 4, pp. 253-261, 2015.

[30] H.G. Park, Y.R. Lee, J.K. Jun, and W.L. Lee, "Exercise training is more effective than resveratrol supplementation on alleviation of inflammation in peritoneal macrophages of high fat diet mice," vol. 18, no. 1 , pp. 79-87, 2014.

[31] J.K. Jun, W.L. Lee, H.G. Park, S.K. Lee, S.H. Jeong, and Y.R. Lee, "Moderate intensity exercise inhibits macrophage infiltration and attenuates adipocyte inflammation in ovariectomized rats," vol. 18, no. 1, pp. 119-127, 2014.

[32] J.H. Jeong, H.G. Park, Y.R. Lee, and W.L. Lee, "Moderate exercise training is more effective than resveratrol supplementation for ameliorating lipid metabolic complication in skeletal muscle of high fat diet-induced obese mice," vol. 19, no. 2, pp. 131-137, 2015.

[33] N. Kawanishi, H. Yano, T. Mizokami, M. Takahashi, E. Oyanagi, and K. Suzuki, "Exercise training attenuates hepatic inflammation, fibrosis and macrophage infiltration during diet induced-obesity in mice," Brain. Behav. Immun., vol. 26, no. 6, pp. 931-941, 2012.

[34] N. Kawanishi, H. Yano, Y. Yokogawa, and K. Suzuki, "Exercise training inhibits inflammation in adipose tissue via both suppression of macrophage infiltration and acceleration of phenotypic switching from M1 to M2 macrophages in high-fat-diet-induced obese mice," Exerc. Immunol. Rev., vol. 16, pp. 105-118, 2010.

[35] W. Zhang, H. Kudo, K. Kawai, S. Fujisaka, I. Usui, T. Sugiyama, K Tsukada, N. Chen, and T. Takahara, "Tumor necrosis factor-?? accelerates apoptosis of steatotic hepatocytes from a murine model of non-alcoholic fatty liver disease," Biochem. Biophys. Res. Commun., vol. 391, no. 4, pp. 1731-1736, 2010.

[36] P.A.M. Cavalcante, M.F. Gregnani, J.S. Henrique, F.H. Ornellas, and R.C. Araújo, "Aerobic but not Resistance Exercise Can Induce Inflammatory Pathways via Toll-Like 2 and 4: a Systematic Review," Sport. Med. - Open, vol. 3, no. 1, 2017.

[37] V. Braunersreuther, G.L. Viviani, F. Mach, and F. Montecucco, "Role of cytokines and chemokines in non-alcoholic fatty liver disease," World J. Gastroenterol., vol. 18, no. 8, pp. 727-735, 2012

[38] M.A.M. Den Boer, P.J. Voshol, J.P. Schröder-van Der Elst, E Korsheninnikova, D.M. Ouwens, F. Kuipers, L.M. Havekes, and J.A. Romijn, "Endogenous interleukin-10 protects against hepatic steatosis but does not improve insulin sensitivity during high-fat feeding in mice," Endocrinology, vol. 147, no. 10, pp. 4553-4558, 2006.

[39] D.E. Cintra, J.R. Pauli, E.P. Araújo, J.C. Moraes, C.T. de Souza, M Milanski, J. Morari, A. Gambero, M.J. Saad, and L.A. Velloso, "Interleukin-10 is a protective factor against diet-induced insulin resistance in liver,” J. Hepatol., vol. 48, no. 4, pp. 628-637, 2008. 